\title{
SALMONELLOSIS INTESTINAL EN EL LACTANTE
}

\author{
por los IYtes. ARTURO SGROG;Gle, HI:MBERTO GARGES. ALFONSO COSTA Y \\ JOSE. ACIIIATT \\ Clinica pediatrica del Prof. A. Scroggic:-Huspital de Niños "Roberto del Rio"
}

Las investigaciones bacteriológicas en el capitulo de las diarreas infantiles, en especial en la edad del lactante, han dado una importancia cada día mayor á las salmonellas como agentes etiológicos de estos cuadros, que en nuestro medio hospitalario han alcanzado at cerca del $25 \%$ del total de las diarreas infecciosas en esta edad de la vida, en el curso de los últimos años. La Jocalización intestinal de estos agentes, con mucho la más frecuente, justifica un estudio esperial de estos cuadros que designaremos con el nombre de Salmonellosis Intestinal y que estudiaremos en este trabajo principalmente bajo el panto de vista clinico y terapéutico.

\section{Acción patógena de las salmonellas en el lactante}

El género Salmonella está constituido por bacilos Gram negativos, de caracteristicas bioquímicas bien delinidas $y$ serológicamente afines. El número de especies de salmonellas ha ido aumentando notablemente en los últimos años: en la última edición del libro de
Bergey (1948) se reconocen $15 \mathrm{l}$ y en el reciente libro de Kaulfmann (1951) alcanzan a 209 las aceptadas oficialmente. Iil reconocimiento de las especies se basa, bajo el punto de vista bacteriológico, en un análisis cuidadoso de là estructura antigésica, debiendo investigarse la presencia de los antigenos somático (O) y tlagelar $(\mathbf{H})$, este último en dos fases. Estos unicroorganismos pueden ser patógenos para el hombre, para los animales o para ambos it la ve\%. Atendiendo a la capacidad de producir enfermedad en la especie humana, se les puede clasificar en 3 grupos:

a) Tipos patógenos absolutos, que siempre dan lugar a cuadros mórbidos en la especie huunana. Entre ellos se incluyen la S. Typhosa (B. de Eberth), la S. Paratyphi (B. Paratífico A), Ia S. Schottmulleri (B. Paratífico B) y la s. Hirschfeldi (B. Paratífico $\mathbf{C}$ ).

b) Tipos patógenos relativos, que comprenden aquellas especies que son patigenas habituales para determinados animales, pero que ocasionalmente pueden también originar enfermedad en la especie humana. En este grujo se encuentran la mayor parte de las especies 
de salmonellas conocidas (S. Typhimuriubu, $\$$ Enteritidis, S. Bovismorbificans, \$. Newport, etc.).

c) Tipos no palógenos, que incluyen aquellas salmonellas que son patógenas úmiómente para ciertas especies animates y que no determinan, por consiguiente, enfermedad en el hombre. Entre cstas polemos citar la S. Abortus equis, la $S$. abortus ovis, la $S$. pollorum, la S. gallinarum, etc.

Debemos destacar, lesde luego, que los organismos jóvenes tienen una mayor receptiviclad para estos gérmenes, de allí que los cuadros intestinales que se encuentran en la práctica se refieran especialınente a lactantes, siendo más raramente observados en niños de segunda infancia. Es un hecho semejante a lo que öcurre con el colibacilo y el proteus, que siendo saprófitos habituales del intestino, pue den ocasionar diversos cuadros patológicos en el rectén nacido y en el lactante menor de 3 meses, por las condiciones inmunitarias propias de esta edad de la vida. De esta observación clinica y de las investigaciones bacteriológicas de los autores uruguayos Hornaeche, Peluffo y Aleppo, ha surgito la llamada Doctrina de Montevideo, que ha tenido gran repercusión en el ambiente cientilico. Según ella, estos gérmenes de origen animal son capaces de determinar enfermedad hunıana, a condición de que lleguen a un organisno joven; hecho negado por la Doctrina de Kiel que, basíndose en la relativa inmunidad del adulto por los gérmemes del género salmonella, les negaba acción jatógena para la especie humana.

La fuente de contagio, al parecer la más importante por lo menos para el lactante, es la interhumana a partir cle otro niño enfermo o adultos portadores y la vía de contagio las manos de la enfermeril o persona que lo cuida. Jos utensilios, el termómetro, las ropas, etc. Ėl rontagio a partir de alimentos de origen animal es muy poco protable tratándose de lac. tantes y tiene importancia únicamente para el adulto o el niño mavor.

El periodo de incubación de la inlección salmonellósica es breve: de 1 a 2 dias en los casos comprobados de intoxicación alimenticia, como así también lo lué en los casos experimentales de Hormaeche.

La salmonellosis puede determinar cantbios bumorales reconocibles por las reacciones de aglutinación especifica, investigables en el suero de Ios enfermos, que tienen gran interés como método de diagıóstico. Sin enıbargo, esta infección no deja inmunidad, ya que se han podido observar reinfecciones varias veces en el mismo niño con la misma especie de salmonella. Lo que sí parece ser evidente es que se desarrolla con la edad una resistencia orgánica adquirida, que se pone en evidencia en la clinica por la mayor benignidad de los cuadros mórbidos producidos por estos agentes y por su menor tendencia a la difusión por vía sanguínea (septicemias, localizaciones a distancia).

El atribuir los cuadros diarreicos observados en la patologia infantil a estos agentes, se justifica por los siguientes hechos de observación dinica:

$1^{9}$ Concordancia entre la existencia de cuadros diarreicos agudos en lactantes y la presencia de salmonellas en el coprocultivo;

20 Mayor trecuencia del hallazgo de salmouellas en las deposiciones en los períodos def año en que se abserva un mayor número de diarreas (époci estival);

39 Comprobación de brotes epidémicos de enterocolitis en lactantes y fenómenos gastrointestinales del tipo de la intoxicación alimenticia en niños mayores y adultos, coincidiendo con el hallazgo de salmonellas en sus deposiciones, en medios con buenas condiciones sanitarias y de higiene (epidemias intrahospitalarias, por ejemplo);

49 Comprobación de adultos o niños mayores portadores de salmonellas en los centros en que se han observado tales brotes epidémicos;

5 \% Hallazgo concordante de salmonellas en la sangre y otros productos patológicos (orina, líquido céfalo-raquídeo, etc.), en los lactantes afectos de diarrea por salmonella, $y$

60 Demostración de la existencia de aglutinislas específicas en el suero de los enfermos afectados, para la ıuisma especie de salmonella encontrada en el coprocultivo.

Debemos reconocer que el hecho de encontrar salmonellas en el cultivo de las deposiciones o de la sangre, to es suliciente argumento para atribuirles un papel patógeno, ya que dichos gérmenes pueden hatlarse como simples saprófítos en el intestino o aún causar hacteremias transitorias en niños con mal estado inmunitario, que no tienen signilicación patologica especifica.

Por lo que respecta a lá comprobación de aghutininas, debemos decir que las opiniones de los autores no concuerdan del todo, ya que la presencia de ellas en el suero de los enfermos es dificil de comprobar y constituye adenás un problema térnico complicado, dado el gran número de especies de salmionellas hoy dia identificadas. Por otra parte, hay que to- 
mal en cuenta que los mecanismos inmunitarios aún incompletamente desarrollados en el lactante, hacen que las reacciones de aglutinación aparezcan tardíamente, siendo posible que las pruebas resulten negativas durante el período agudo de la entermedad, que en ocasiones suele ser muy corto.

\section{Clínica y Anatomia Patológica de la Salmonellosis Intestinal}

Las salunonellas puedeu originar en la especie humana, en particular en el lactante menor, tanto cuadros en los que has manifestaciones gastrointestinales son las predominantes, como tambićn sepsis o localizaciones variadas en órganos alejados del tubo digestivo (meningitis, pleuresias, artritis, etc.). Lát Salmonellosis Intestinal, que es la formá más frecuente, puede dar lugar a 2 sinctromas bien definidos: a un cuadro diarreico agudo, con aspecto enterocólico o simplemente dispéptico, sin septicemia; o bien, a un cáarlro tífico, con septicemia, alteración sensorial, fiebre alta $y$ persistente, hepato y espletiomegalia, que puede dar or $i^{-}$ gen a localizaciones extraintestinales a distan. cia. La presencia de una u otra cle estas formas clínicas depende de la edad del niño, del agente en juego, de la virulencia del germen, de la masividad del contagio y del grado de desarrollo de los mecanismos defensivos del paciente. Fuera de estos aspectos, tiene tambićn importancia, a nuestro juicio, la coexistencia de infección simultánea de salmonellas con otros agentes patógenos intestinales que pue. den hacer variar su virulencia.

En este trabajo nos releriremos exclusivamente a las salmonellas de origen animal, que llegadas al tubo digestivo del Jactante pueden provocar alteraciones inllamatorias que se manifiestan predominantemente por el síntoma diarrea. Excluimos, por consiguiente, los casos con cuadro tifico, las septicemias y las Iocalizaciones extraintestinales producidas por estos mismos agentes.

En la casi totalidacl de nuestros enfermos hemos observado un cuadro diarreico agudo de intensidad mediana o grave, que puede presentarse bajo el aspecto de una enterocolitis disenteriforme o como una dispepsia simple alimenticia. En la printera forma hay cleposiciones mucasanguinolentas o mucopiosanguinolentas, reflejo gastro-cólico, dolores abdominales y tenesmo rectal; en la segunda forma observamos deposiciones semiliquidas a disgregadas, con o sin mucus, inapetencia y vómi- tos alimenticios, durante los primeros dias. Tanto una como la otra forma de diarrea puede dar origen a un sindroma tóxico con gran deshidratación, alteración sensorial, colapso vascular, trastorno metabólico y acidosis.

Estos cuadros diarreicos son imposibles de diferenciar clínicamente de las diarreas inespecíficas o de las disenterias bacilar o amebiand, siendo el examen bacteriológico y parasitológico de las deposiciones el único nedio seguro de diagnóstico dilerencial. En todo caso, siempre nos quedará Ia duda de si el cuadro diarreico existente es realmente producido por las salmonellas encontratas en las deposiciones o si el hallazgo de ellas es un hecho sin relación de causa a efecto con la diarrea, siendo tales lactantes simplemente portadores de estos agentes que pulutan en sui intestino en calidad de saprófitos. En la solución de este punto cobra toda su importancia la investigación de las aglutininas específicas en el suero de los enfermos.

Otro hecho incierto de la Sitnonellosis Intestinal es el referente a las lesiones anátomopatológicas que ella determina. En la literatura se encuentran descritos casos de niños fallecidos por diarrea, con hallazgo de salmonellas en Ias deposiciones, en cuyas autopsias se encontraron lesiones inflamatorias de la pared intestinal que en nada se diferencian de Ia enterocolitis folicular descrita por Wiederhoffer. En otras palabras, no se han descrito lesiones anatómicas específicas propias de este tipo de infección $\mathrm{y}$, aun más, en muchos casos no hay evidencia de ellas. En nuestra casuistica tenemos el ejemplo de un lactante fallecido en el período agudo de la diarrea, $c$ n el cual el anátomo-patólogo no encontró ninguna alteración morfológica de la mucosa in testinal. Referente a la existencia de lesiones microscopicas, hay que tener uncha cautelit en su interpretación, por cuanto se producen alteraciones cadavericas de la pared del intestino, ya a las pocas horas de producido el deceso, que pueden inducirnos a error.

La gravedad de la Salmonellosis Intestinal en el lactante está elt relación inversa con $1 a$ edad del niño, hccho explicable porque sus mecanismos defensivos estún menos desarrollados. La mortalidad, a pesar de ello, ha llegado a ser muy baja desde que contamos con antibióticos adecuados, como lo pondremos en evidencia más adelante.

Finalmente debemos recakar la posibilidad del hallazgo de salmonellas en las deposiciones de lactantes que han padecicto anterior- 
mente de uno o más cuadros diarreiços y aún en niños que nunca han tenido manitestaciones gastrointestinales. Se trata de portadores convalecientes o sanos, respectivamente, que pueden ser la fuente de contagio en los brotes epidémicos observados en Servicios de Lactantes en varios países.

\section{Consideraciones generales sobre nuestros entermos}

Nuestro material clinico ha sido recopilado en el curso de un año (octubre de 1950 a septiembre de 1951, anbos inclusives) y se refiere a 113 lactantes que padecían de cuadros diarreicos agudos y cuyo coprocultivo puso en evidencia la presencia de salmonellas. Este número de enfermos, que a primera vista puede parecer extraordinariamente numeroso para nuestro medio, puede explicarse por el hecho de haberse practicado una investigacion sistemática y repetida de las deposiciones de aquellos lactantes que consultaban al Hospital por diarreas agudas. Para efectuar esta investigación, se organizó un Servicio especial en el Consultorio Exterio de Lactantes, destinado exclusivamente a la investigación de la etiología de las diarreas agudas. Los niños eran enviados directamente a1 Laboratorio, donde se tomaba muestra de las deposiciones mediante la introducción de una sonda rectal conectada con un frasco estéril, que era examinada inmediatamente al fresco para la investigación parasitológica y además era sembrada en los medios de cultivo adecuados para la investigación bacteriológica. En otros casos tomábamos muestra directa por rectoscopia, que era enviada rápidamente al Laboratorio para efectuar estas investigaciones. Los exámenes coprológicos eran repetidos diariamente o día por medio hasta un número de 5 por cada entermo, según la gravedad y duración del cuadro clinico. Los casos medianos y leves eran tratados en el Consultorio Externo; en tanto que los graves o acompañados de sindrome tóxico fueron hospitalizados para su mejor estudio y tratamiento.

$$
\text { Cuadro Xro } 1
$$

DISTRIBUGCION DE LOS CASOS SHRAN L.A F.DAD

\section{Edades:}

0 a $\$$ mess

3 a 6 meses

6 a 12 unescs

l a $21 / 2$ años

Iotal:
Ne de casos:

$\left.\begin{array}{l}28 \\ 17 \\ \mathbf{3 5} \\ \mathbf{3 3}\end{array}\right\} 70 \%$

En el cuadro $\mathrm{N}^{9}$ l se ordenan los enfermos según sus edades, las que fluctuaron entre el período del recién nacida y los $21 / 2$ años. Puede verse que el $70 \%$ de los casos se observó en niños menores de un año, hecho que confirma, una vez más, lo que ya se ha comprobado en el extranjero. Entre los menores de tres meses se incluyen 11 lactantes menores de un mes $(25 \%$ de este grupo) de los cuales 6 tueron niños prematuros de la Sala de Incubadoras; en estos 11 recićn nacidos se epcontraron las siguientes especies de salmonellas: Bareilly 1 caso, Derby 2 casos, London 1 caso, Newport 2 casos y Typhimurium 5 casos. Ls llamativo el hecho de que entre los recien nacidos en casi Ia mital de los casos se encontrala la S. Typhimurium y que ellos se obser. varan en un corto período de tiempo, to que nos hace pensar que se trató de un contagio intrahospitalario. El cuadro clínico fué en general tanto nás grave cuanto menor edad tenia el ninoo, lo que está en relación con el menor desarrollo de sus mecanismos defensiros.

$$
\text { Cuadro No } 2
$$

DISIRIBUEION DE LOS CASOS SEGLN" EL ESTADO NLTRITIVO

Estado nutritivo
Eutrofia
- Distrofia simple
Distrofia avanzada
Atrofia
Prematuros
Total:

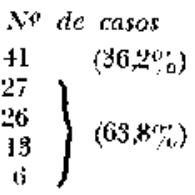

113

En el cuadro No 2 se distribuyen los casos según el estado nutritivo de los enfermos. Se catalogaron como Distrofia simple los que tenian un déficit de peso hasta de un $25 \%$; como Distrofia avanzada los que tenían entre un 25 y un $50 \%$ de déficit ponderal y como Atrofia aquellos a los cuales faltaba más del $50 \%$ deI peso normal para su edad. Como Prematuro se clasificaron los menores de un mes con peso de nacimiento inferior a $2.500 \mathrm{grs}$. Entre los distróficos graves y los atróficos había 7 niños con cuadro policarencial con edemas. Se puede ver que solamente un poco más de un tercio de los enfermos $(36,2 \%)$ eran niños eutróficos, el resto $(63,8 \%)$ tenían un trastorno nutritivo crónico más a menos intenso. No hemos podido comprobar que el estado nutritivo tenga importamcia determinante o predisponente en la infección del lactante por salmonellas; pero, eso sí, la tiene y en forma evidente, bajo el punto de vista clínico, en la duración 
de la enfermedad que determinan, caracterictica de la disergia propia del estado distrófico.

$$
\text { Cundra N.* } 3
$$

\section{ESPECIES DE SALMONELLAS AISLADAS EN LOS COPRO-} Cultivos

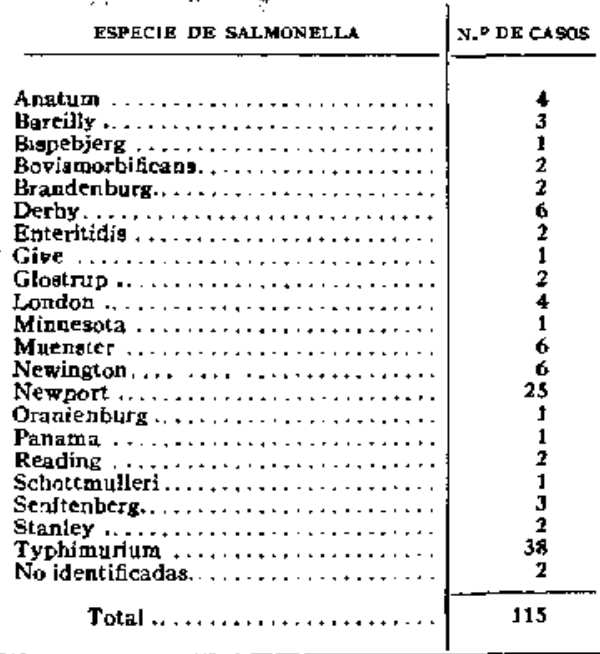

En el cuadro No 3 se indican los hallazgos bacteriológicos encontrados en nuestros 113 enfermos, ordenados alfabéticamente por el nombre de la especie de salmonella aislada en el coprocultivo. En total se identificaron 21 especies diversas. Se puede ver que la S. Typhimurium $(33 \%)$ y la $\mathrm{S}$. Newport $(21,7 \%)$ son los dos agentes más frecuentes en nuestro medio; le siguen en orden de frecuencia la $\mathbf{S}$. Derby, la S. Muenster y la S. Newington (con $5,2 \%$ cada una) y luego la $\mathrm{S}$. Anatum y la S. London (con $3,4 \%$ cada una). Debemos aclarar que la diferencia entre el número de especies de salmonellas aisladas y el número de enfermos, se debe a que en un caso el coprocultivo demostró infección sucesiva de 2 especies distintas de salmonellas: S. Stanley y $\mathrm{S}$. Derby y otro caso era un portador simultáneo de S. Anatum y S. Muenster. En sólo 2 casos no fué posible identificar la especie de salmonella hallada en el coprocultivo.

$$
\text { Cuadro n.o } 4
$$

DISTRIBUCION DE LOS CASOS SEGUN EL CUADRO CLINICO Y LOS TIPOS MAS FRECUENTES DE SALMONELLA

\begin{tabular}{|c|c|c|c|c|c|c|}
\hline \multirow{2}{*}{$\begin{array}{c}\text { THPO DE SALGONELLA } \\
\text { AISLADO }\end{array}$} & \multicolumn{2}{|c|}{ D. disenterif. } & \multicolumn{2}{|c|}{ D. digpeptic. } & \multirow{2}{*}{$\begin{array}{l}\text { Sin } \\
\text { dia- } \\
\text { rrea }\end{array}$} & \multirow{2}{*}{$\begin{array}{l}\text { Núm. } \\
\text { de } \\
\text { casog }\end{array}$} \\
\hline & cltox & s!tox & c|lon $x$ & $\mathrm{~s} \mid \operatorname{tox}$ & & \\
\hline $\begin{array}{l}\text { Typhimurium } \ldots \ldots \ldots \\
\text { Newport } \ldots \ldots \ldots \ldots \ldots \\
\text { Oters tipos } \ldots \ldots \ldots \ldots \ldots\end{array}$ & $\begin{array}{l}6 \\
0 \\
4\end{array}$ & $\begin{array}{l}17 \\
16 \\
18\end{array}$ & $\begin{array}{l}4 \\
2 \\
5\end{array}$ & $\begin{array}{r}8 \\
6 \\
19\end{array}$ & $\begin{array}{l}3 \\
1 \\
4\end{array}$ & $\begin{array}{l}38 \\
25 \\
30\end{array}$ \\
\hline Totales......... & 10 & 51 & 11 & 33 & 8 & 113 \\
\hline
\end{tabular}
AJSLADOS EN EL, COPROGULTIVO
El cuadro No 4 ha sido confeccionado con el objeto de estudiar una posible relación entre el cuadro clínico que presentaban los enfermos y las especies de salmonellas aisladas de las deposiciones. Solamente los casos producidos por $\mathbf{S}$. Typhimurium y $\mathrm{S}$. Newport son susceptiblés de comparación, por el mayor número de casos de cada grupo; las restantes especies de salmonelląs las tomamos en conjunto ya que el pequeño número de enfermos de cada una de ellas no permite compararlas individualmente. Hemos considerado dos tipos fundamentales de diarreas: el tipo disenteriforme y el tipo dispéptico, de acuerdo con las características ya enunciadas anteriormente y subdividido cada uno de estos grupos en formas con y $\sin$ toxicosis. De su estudio en conjunto, es posible deducir que la forma disentérica $(54,2 \%)$ es más frecuente que la dispéptica $(38,8 \%)$. En 8 enfermos $(7 \%)$ no había diarrea en el momento de ingresar al Servicio, pero tenian antecedentes de haber padecido de diarrea aguda con anterioridad. lo que permite calificarlos como portadores convalecientes, con mucha probabilidad. En estos 8 enfermos se identificaron las siguientes especies de salmonellas: 1 caso con S. Anatum, 2 casos con S. Newington, I caso con S. Newport, 3 casos con $S$. Typhimurium y 1 portador de dos especies diferentes: $S$. Anatum y $S$. Muenster.

$$
\text { Cuadro N.o } 3
$$

\begin{tabular}{|c|c|c|c|c|}
\hline $\begin{array}{l}\text { ESPECIE } \\
\text { DE SALMONELI.A }\end{array}$ & $\begin{array}{c}\text { Con } \\
\text { Loxicosis }\end{array}$ & $\begin{array}{c}\text { Sin } \\
\text { toxicosis }\end{array}$ & $\begin{array}{l}\text { Nam. de } \\
\text { casos }\end{array}$ & $\operatorname{con} \%$ \\
\hline $\begin{array}{l}\text { Typbimutium } \ldots \ldots \\
\text { Newnort } \ldots \ldots \ldots \ldots \\
\text { Otros tipos } \ldots \ldots \ldots\end{array}$ & $\begin{array}{r}10 \\
2 \\
9\end{array}$ & $\begin{array}{l}28 \\
23 \\
41\end{array}$ & $\begin{array}{l}38 \\
25 \\
50\end{array}$ & $\begin{array}{r}26,3 \\
8,0\end{array}$ \\
\hline Totales........ & 21 & 92 & 113 & 18,5 \\
\hline
\end{tabular}

ESTUDIO COMPARATJVO DE LOS CASOS CON TOXICOSIS EN LOS 113 ENFERMOS

En el cuadro No 5 se estudia la relación en. tre la existencia de una sindroma tóxico y las especies de salmonellas más frecuentes. Podemos ver que la $S$. Typhimurium da un alto porcentaje de toxicosis: 26,3\% (10 casos en $38)$, en comparación con la $\mathrm{S}$. Newport que sólo da un $8 \%$ (2 casos en 25). El otro rubro del cuadro, no es susceptible de ser comparado en igual forma, por incluir 19 especies diferentes de salmonellas en los 50 casos. En total en los $11 \dot{3}$ enfermos hubo 21 con toxicosis $(18,5 \%)$. 
Cu*deo N.* G

HALLAZGO DE SALMONELLAS SIMULTANEAMENTE CON OTROS AGENTES PRODUCTORES DE DIARREA AGUDA EN 113 CASOS

\begin{tabular}{|c|c|}
\hline RESULTADO DEL EX, DI DEPOSICIONES & N.a de casos \\
\hline 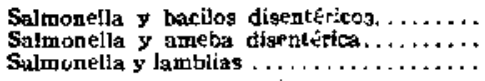 & $\begin{array}{r}5 \\
1 \\
14\end{array}$ \\
\hline Total $\ldots \ldots \ldots \ldots \ldots+\ldots \ldots \ldots \ldots$ & 20 \\
\hline
\end{tabular}

En el cuadro No 6 se expone el hallazgo simultáneo de salmonellas con otros agentes productores de diarreas en el niño, en los 113 casos. En él puede verse la alta frecuencia de la asociación de lamblia y salmonella, lo que está en relación con la gran difusión de la infestación lambliásica en nuestro medio.

\section{Resultado del tratamiento con antibióticos}

Para el tratamiento antibiótico de nuestros enfermos, hemos empleado la Estreptomicina, la Cloromicetina y la Aureomicina.

La Estreptomicina la usamos por vía oral, disuelta en suero fisjológico, en dosis de 500 mgrs. diarios en los menores de 6 meses y 1 gr. diario en los mayores de esta edad, fraccionada cada 2, 3 o 4 horas con las tomas de alimento.

La Cloromicetina se empleó en dosis de $\mathbf{5 0}$ a $\mathrm{I00}$ mgrs. diarios por $\mathrm{Kg}$-peso, según la gravedad del cuadro clínico, fraccionándola cada 4 a 6 horas. Este antibiótico fuć en general bien tolerado, aungue troperamos con el inconveniente de su mal sabor, que suele producir vómitos: con la preparación de cúpsulas pequeñas conteniendo 25 a $50 \mathrm{mgrs}$ y posteriormente usando el Palmitato de Cloromicetina de Parké, Davis y Co., la tolerancia fuć perfecta.

La Aureomicina la indicamos en dosis diarias de 25 mgrs. por $\mathrm{Kg}$-peso, Iraccionándola en tomas cada 4 a 6 horas. $\Lambda$ pesar de su mal sabor, fué bien tolerada en aquellos lactantes, que no pudiendo ingerir las cápsalas de 50 mgrs., les era dada disuelta en agua edulcorada con sacarina.

En nuestros enfermos, en general, iniciamos el tratamiento con Estreptomicina oral antes le conocer el resultado de la investigación bacteriológica. Conocido el resultado positivo para salmonellas del coprocultivo, continuábamos este tratamiento por $8 \cdot$ a 15 dias, si el resultado clínico era favorable. En casos con mejoría transitoria y luego recaída, repetiamos una segunda cura de Estreptomicina y cuando habia fracaso la reemplazábamos por Cloromicetina en curas de 10 a 15 dias. En 20 casos, sin embargo, en los que el estado del niño permitió esperar el informe bacteriologico de las deposiciones, se emple $\delta$ la Cloromicetina como primer tratamiento: La Aureomicina, en curas de 10 dias, la usamos solamente en 5 enfermos: en 1 después del fracaso de la Cloromicetina y en 4 que no habian recibido tratamiento antibiótico previo.

Los resultados observados con estas curas de antibióticos, las estudiaremos detalladamente en seguida.

$$
\text { Cagdta } N \text {, , }
$$

RESULTADD DEL, TRATAMIENTO CON ESTREPTOMICINA ORAL EN g9 GASOS. DISTRIBUDDOS SEGUN BL CUADRO CLINICO DE LOS ENFERMOS

\begin{tabular}{|c|c|c|c|c|c|c|}
\hline \multirow{2}{*}{ BESULTADO } & \multicolumn{2}{|c|}{ D. disenterif. } & \multicolumn{2}{|c|}{ D. dispeptic. } & \multirow{2}{*}{$\begin{array}{c}\text { Nám. } \\
\text { de } \\
\text { cugos }\end{array}$} & \multirow{2}{*}{$\begin{array}{c}\text { Porcen- } \\
\text { tizje }\end{array}$} \\
\hline & c|tox & etox & c|tox & e|tor & & \\
\hline $\begin{array}{l}\text { Curacion sin recaida } \\
\text { Curac. con recalda - } \\
\text { Curac. con persistes. }\end{array}$ & $\begin{array}{l}1 \\
\mathbf{1}\end{array}$ & $\begin{array}{r}11 \\
3\end{array}$ & $\begin{array}{l}3 \\
2\end{array}$ & $\begin{array}{r}12 \\
5\end{array}$ & $\begin{array}{l}27 \\
11\end{array}$ & $\begin{array}{l}30.3 \\
12.3\end{array}$ \\
\hline $\begin{array}{l}\text { cla del germen ... } \\
\text { Fracaso } \ldots . . . \ldots \ldots \\
\text { Fadlecidos ......... }\end{array}$ & $\begin{array}{l}1 \\
3 \\
0\end{array}$ & $\begin{array}{r}5 \\
12 \\
1\end{array}$ & $\begin{array}{l}5 \\
2 \\
0\end{array}$ & $\begin{array}{r}6 \\
15 \\
1\end{array}$ & $\begin{array}{r}17 \\
32 \\
2\end{array}$ & $\begin{array}{r}19,1 \\
35,9 \\
2,2\end{array}$ \\
\hline Totales......... & 6 & 32 & 12 & 39 & 89 & $99,8 \%$ \\
\hline
\end{tabular}

El cuadro $N^{0} 7$ se refiere al tratamiento con Estreptomicina por vía oral de 89 enfermos, clasificados de acuerdo con el cuadro clínico que presentaban. Se considera curación $\sin$ reraida los que mejoraron clínica y bacteriológicamente con l sola cura de Estreptomicina y curación con recaida aquellos enfermos que después de una mejoría transitoria recayeron de la diarrea y curaron definitivamente con sucesivas curas de Estreptomicina (2 o 3). Llamamos curación con persistencia del germen aquellos casos en los que la mejoria clinica no fué paralela con la desaparición de las salmonellas de las deposiciones o, dicho de otro moclo, aquellos enfermos que rípidamente mejoraron de la diarrea pero cuyo coprocultivo continuó siendo positivo por un tiempo más a menos largo y que requirieron nuevas curas de antibióticos para hacerlas desaparecer por completo. Se consideran fracasos del tratamiento los que no mejoraron ni clinica, ni bacteriológicamente con las curas del antibiótico.

De los 89 casos curaron sin recaída 27 $(30,3 \%) ; 12$ de la forma disenteriforme, de los cuales 1 con toxicosis y 15 de Ia forma dispéptica, de Jos cuales 3 con toxicosis.

Tuvieron curación con recaída 11 (12,3\%); 4 de la forma disenteriforme, de los cuales ] 
con toxicosis y 7 de la format dispéptica, de los cuales 2 con toxicosis.

Curaron con persistencia del germen 17 $(19,1 \%) ; 6$ de la forma disenteriforme, de los cuales 1 con toxicosis y 11 de la Lorma dispéptica, de los cuales 5 con toxicosis.

E1 tratamiento con Estreptomicina fracasó en 32 casos $(35,9 \%) ; 15$ de la forna disenteriforme, de los cuales 3 con toxicosis y 17 de la forma dispeptica, de los cuates 2 con toxicosis.

De los 89 carsos fallecicron 2 (2,20\%). Ln uno, se trataba de un. lactante distrólico de 3 meses de edad, que consultó por una diarrea disenteriforme, encontrándose $S$. 'Typhimurium en el coprocultivo. Fué tratarlo con Estreptonnicina oral ( $1 / 2$ gr. diario por 6 tlas) con mejoría clímica rápida. Deja de concurrir a la Consulta Externa y 15 días más tarde recie de la diarrea, acompañada de un estado infeccioso grave, de lo cuat fallece a las 24 horas. No se bizo autopsia por ser un caso ratado en la Consulta Externa.

El otro fallecido era un lactante de un año de edad con una Atrofia Policarencial con edemas y diarrea 'dispéptica it repetición que se trató con Estreptomicina oral (l gr. diario por $5 \frac{1}{2}$ días) sin modificarse la diarrea y con baja de peso progresivo. En el coprocultivo se halló la S. Newport. Falleció al $7^{\circ}$ día de hospitalización, encontrándose en la autopsia: "Atrolia, Bronconeumonia y Anemia". No habia lesiones intestinales.

En resumen, de los 89 casos tratados con Estreptomicina oral mejoraton 55, luubo fracaso en 32 y tallecen 2 . El porcentaje de curaciones fuc de $6 \mathrm{I}, 79 \%$, el de fracaso $35,95 \%$ y Ia letalidad de $2,24 \%$.

$$
\text { Cundron n.os }
$$

RESULTAUO DEL TRATAMIENTO CON GLOKOMICETINA EN 52 CASOS DISTRIBUUDOS SEGUN EL CUATRO CLINICO DE LOS ENFERMOS

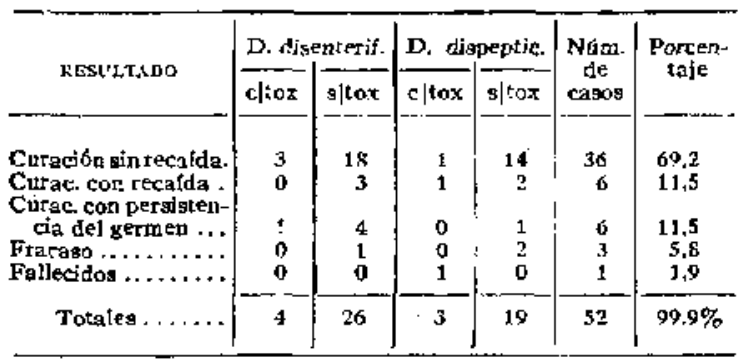

En el cuadro No 8 se resume el resultado del tratamiento con Cloromicetina, siguiendo el mismo ordenamiento que en los casos tratados con Estreptomicina, pasa poder estable- cer comparaciones. Para su interpretación son también válidas las explicaciones ya dadas al referirnos al cuadro $\mathrm{N}^{0} 7$ :

En 32 enfermos se usó la Cloronicetina des. pués del fracaso de la Estreptomicina y en 20 enfermos que no habian recibido tratamiento antibiótico previo.

De los 52 casos, 36 curaron sin recaida $(69,2 \%), 2 l$ de la forma disenteriforme, de los cuales 3 con toxicosis y 15 de la forma dispép. tica, de los cuales 1 con toxicosis.

Curaron con recaida 6 enfermos $(11,5 \%)$; 3 de la forma disenteriforme sin toxicosis y $\mathbf{3}$ de la lorma dispéptica. de los cuales 1 con toxicosis.

Hubo curación con persistencia del germen en 6 casos $(11,5 \%) ; 5$ de la forma disenteriforme, de los cuales 1 con toxicosis y 1 de la Cormá dispéptica sin toxicosis.

Fracasó el tratamiento en 3 casos $(5,8 \%)$; 1 de la forma disenteriforme y 2 de la forma dispéptica, todos ellos sin toxicosis.

Falleció 1 caso $(1,9 \%)$. Se tratabá de un lactante de 2 meses que ingresa con Atrofia $y$ Toxicosis. Tenia deposiciones líquidas mucosas frecuentes, encontrándose en el coprocultivo la $S$. Enteritidis. Como tratamiento se le hizo fleboclisis, plasmoterapia, alimentación con suero de babeurre y crema de arroz y Estreptomicina oral ( $1 / 2$ gr. diario por $51 / 2$ días). A pesar de ello, el cuadro diarreico se agrava, llegando Ia frecuencia de las deposiciones hasta 18 en el día y apareciendo tenesmo rectal. Se cambia el tratamiento de Cloromicetina (60 mgrs. por Kg-peso al dia por 13 días), con lo que únicamente se logra disminuir algo la frecuencia de las deposiciones. Fallece a los 19 dias de estada. En Ia autopsia se encontró una Enterocolitis ulcerosa del tipo de disentería bacilar y una Peritonitis fibrinosa. Este caso, el único fallecido de este grupo, merece un comentario especial. Se trató posiblemente de una infección mixta por salmonella y bacilo disentérico; aunque éstos no aparecieron en el coprocultivo, las lesiones anátomo-patológicas del intestino permiten afirmar este diagnóstico, lo que explica el fracaso del tratamiento antibiótico con Estreptomicina y Cloromicetina. Este lactante no recibió sulfoterapia.

En resumen, mejoraron con Cloromicetina 48 de los 52 casos tratados, hubo fracaso en 3 enfermos y fallece sólo 1 caso. El porcentaje de mejoria fué de $92,30 \%$, el de fracaso, $5,76 \%$ y la letalidad de $1,93 \%$.

De los $\mathbf{3}$ casos en que fracasó la Cloromicetina, 2 mejoraron posteriormente con una se- 
gunda cura de Estreptomicina y el otro con una cura de Aureomicina.

Finalmente nos ocuparemos muy brevemente de los 5 casos tratados con Aureomicina. El escaso númeró de enfermos, no nos permite sacar conclusiones y nos referiremos a ellos sin comentario. Uno de los tratados lo fué después del fracaso de la Cloromicetina y los cuatro enfermos restantes no habían recibido tratamiento antibiótico previo; de estos últimos uno tenía, además de una $\mathrm{S}$. Derby, una ameba disentérica.

El resultado del tratamiento de estos $5 \mathrm{cn}$ fermos fuc el siguiente: mejoraron sin recaída 4 casos (incluso el que tenia disentería amebiana, que también mejoró de su amebiasis). Hubo fracaso en l caso, cuyo coprocultivo fué positivo para la $S$. Muenster, que mejoró posteriormente con Cloromicetina.

\section{v. Resumen $\mathbf{y}$ conclusiones}

Se expone la experiencia sobre Salmonellosis Intestinal recogida en el curso de 1 año en el Hospital de Niños Roberto del Río. Se encontraron 113 lactantes que padecian de una diarrea aguda, en los cuales el coprocultivo puso en evidencia la existencia de salmonellas.

La Salmonellosis Intestinal puede producir dos cuadros clínicos bien definidos: un sindroma tffico, con septicemia y un cuadro diarreico agudo $\sin$ septicemia. En la casi totalidad de nuestros enfermos observamos una diarrea aguda de intensidad mediana o grave, que se presentaba bajo los aspectos de una enterocolitis disenteriforme o de una dispepsia simple alimenticia. Ambos tipos de diarrea pueden ori. ginar el sindroma tóxico. Este cuadro es imposible de diferenciar clínicamente de la diarrea aguda inespecifica o de las disenterias bacilar o amebiana, siendo el examen bacteriológico y parasitológico de las deposiciones el único medio seguro de diagnóstico diferencial.

Nos parece que el rol patógeno de estos gérmenes, para la mayor parte de nuestros casos, es evidente. Sin embargo, no podemos desconocer que en una parte de ellos tal hecho resulta dudoso, puesto que la desaparición del agente de las deposiciones no guardó paralelismo con la mejoría de la diarrea, siendo posible que en tales enfermos la salmonella fuera solamente un saprófito intestinal y que la diarrea aguda tuviera una causa alimenticia $o$ infecciosa de otra etiología. Por ello, se hace necesario perfeccionar la investigación de las aglutininas específicas en el suero de los pa- cientes, a fin de poder valorar mejor su verdadero papel patógeno.

Las edades de nuestros enfermos van desde el período del recién nacido hasta los 2 años y medio, siendo el $70 \%$ lactantes menores de un año. En fulestra casuistica se incluyen 11 lactantes menores de 1 mes, $i$ de los cuirles ean prematuros.

Solamente el $36,2 \%$ de nuestrus patientes cran eutroficos: el resto eran niños que padecian de una alteración nutritiva crónica más o menos intensa, incluso 7 con Mistrofia policarencial con edemits.

La gravedid de la enlermedad guarda relaciñn inversa con la edad del enfermo y su evolución es más prolongada, aunque no más severa, en los lactantes afectos de un trastorno nutritivo crónico.

En nuestros 113 casos fueron aisladas 21 especies diferentes de salmonellas, siendo la $\mathbf{S}$. Typhimurium con un $39 \%$ y la $S$. Newport con un $21,7 \%$ los dos tipos más frecuentes.

La diarrea de tipo disenteriforme se observó con mayot frecuencia que Ia de tipo dispéptico: $54,2 \%$ y $38,8 \%$ respectivamente. Hubo un $7 \%$ de niños que no padecian de diarrea aguda en el momento de su estuclio, pero, eso si, tenían antecedentes de haberia padecido con anterioridad, lo. que nos permite clasificarlos con mucha probabilidad como portadores convalecientes.

La toxicosis se obsexvó en el 18,5\% del total de enfermos, siendo la $\mathrm{S}$. Typhimurium la que dió un mayor porcentaje de sindromas tóxicos (26,3\% de este grupo).

En 20 enfermos encontramos, además de Ia salmonella, otros agentes productores de diarrea aguda específica, siendo la laniblia el más frecuentemente hallado, lo que está en relación con el alto indice de infiestación por este protozoo en nuestro medio.

Todos los enfermos fueron sometidos a un esquema dietético uniforme, de acuerdo con la gravedad del cuadro clínico, a lo cual se agregó un antibiótico: Estreptomicina, Cloromicetina o Aureomicina. La Estreptomicina por via oral dió porcentaje de $61,7 \%$ de curaciones, $35,9 \%$ de fracasos y $2,2 \%$ de letalidad. Con la Cloromicetina obtuvimos $92,3 \%$ de curaciones, $5,7 \%$ de fracasos y $1,9 \%$ de mortalidad. La Aureomicina fué empleada en muy pocos casos, to que nos impide sacar conclusiones acerca de su efectividad terapéutica.

Como conclusión final podemos decir que la Salmonellosis es una causa frecuente de diarrea aguda del lactante en nuestro medio 
y que el pronóstico de esta afección es benigno con los antibióticos. actualmente en uso, siendo la Cloromicetina el que nos dió los mejores resultados.

\section{Summary}

The authors report their experience with 113 cases of acute diarrheat caused by salmo. nella, seen at the Hospital Roberto del Rin in the course of one year.

Intestinal salmonellosis an produce two well defined clinical pictures: a typhoid like syndrome with septicemia and an acute: diarrhea without septicemia. In practically all our patients we olserved an acute diarrhea of moderate or severe intensity, that presented itself as a dysenterilorm enterocolitis or as a simple acute nutritional disturbance. Both these types of diarrhea can produce the toxic syndrome. The diagnosis camnot be made clinically and only the stool culture or the parasitologic examination will differenciate it from an acute diarrheil or bacilar or amebic dysentery.

We believe that the pathogenic rol of these organisms in most ol our cases is evident. Nevertheless we camot clisclaim the fact that in some cases the disapearence of the organism from the stools does not correspond with improvement of the diarrhea and in these patients it is possible that the salmonella is only a saprofitic organism, the diarrhea being due to either an infectious or feeding problem. Thuswise it is necessary to insist on studying the specific aglutinins in these patients serum. so as to evaluate there true pathogenic role.

Our patients range in age between newborns and two and a half years, $70 \%$ are under I year of age, of these 11 are under 1 month of age and of these 6 were prematures.

Only $36,2 \%$ of our patients were eutrophic, the rest of them had a chronic nutritional disturbance, 7 of these with multiple defiency with edemas.

The severity of the condition is in inverse ratio to the age of the patient, and the course of the disease is longer although not more severe in those infants with a chronic nutri. tional deficiency.

In our 113 cases we isolated 21 different types of salmonellas; those found most often, were S. Typhimurium, in $33 \%$ and S. Newport in $21,7 \%$ of the cases.

The dysenteriform type of diarrluea was observed in $54,2 \%$ against $38,8 \%$ of the cases in which the diarrhea adopted the caracteristics of an acute nutritional clisturbance. $7 \%$ of the children, although they did not have a diarrhea during their hospital stay. had diarrhea before itrmitanir, sn the most probably were carriers.

The toxic syndrome was present in $18,5 \%$ of the total of our patients, the typhimurium cases giving this syndrome in at higher percentage, $26,3 \%$.

In 20 patients we found besides the salmonetla other agents that can produce diarrhed the commonest among these being the lamblia which is in accordance with the high index of infectivity with this protozoon.

All patients were treated with the same dietetic measures associated to an antibiotic: Streptomycin, Cloromycetin or Aureomycin.

Oral streptomycin gave $61,7 \%$ of cures, $35,9 \%$ of failures and $2,2 \%$ of deaths.

Cloromycetin gave $92,3^{\circ}$ cures, $5,7 \%$ fajlures and $1,9 \%$ deaths.

Aureomycin was used only in a few cases.

In summary we can conclude that in our country, Salmonella is a common case of icute diarrhea among infants, that due to the antibiotics the prognos:s is good, and that among these drugs, cloronycetin we obtained the best results.

\section{B I B L I O G RA F I A}

1.-Bergey's manual of determinative bacteriology. $6^{\text {a }}$ ed. Williams y Wilkins. BaItimore, 1948.

2.-BONABA. J., CARRAU, A., HORMAECHE, F., ZERDINO. V., ALEPPO; P. L., PELCFFO, C. A., atc.: "Estudios sobre la etiología infecciosa de las diarreas infantiles". Ed. J. Garcia Morales. Montcvideo, 1940.

3,-KAUFFAMNN, F. "Enterobactoriaceac". E. Munks. gaard Publisher. Copenhagen, 1951. 